\title{
Anaesthetic Management of a case of Cesarean Section with Peripartum Cardiomyopathy
}

\author{
Ayaz Farooqi, M.D., Waqar-ul-Nisa, M.D., Irshad Ahmad, M.D. \\ Department of Anaesthesiology \& Critical Care, Sher-i-Kashmir Institute of Medical Sciences, Srinagar
}

\section{A B S T R A C T}

\begin{abstract}
We report a case of 28 year old female presenting for an emergency cesarean section with an established diagnosis of peripartum cardiomopathy. Surgery was successfully accomplished under epidural anaesthesia using $15 \mathrm{ml}$. of $0.5 \%$ Bupivacaine, administered in increments. Patient was monitored intra-operatively with Noninvasive blood pressure, pulse oximetry and continuous ECG. Patient's peri-operative course was uneventful. Therefore, it is concluded that in patients with peripartum cardiomyopathy, coming for cesarean section, epidural anaesthesia administered in increments is a safe, acceptable and a better anaesthetic option. JMS 2012;15(1):69-71.
\end{abstract}

Key Words: Cardiomyopathy, epidural, cesarean section, anaesthesia.

Peripartum cardiomyopathy is a relatively rare but life threatening disease. ${ }^{1,2,3}$ It is described as the development of heart failure of unknown etiology occurring during pregnancy or within five months of postpartum period. ${ }^{1,2,3}$ It has an incidence of 1:1300 to 1:4000 live births with an overall mortality rate of $25-30 \% .{ }^{4}$ Mortality and morbidity rates can be higher in subsequent pregnancies if left ventricular dimensions have not been normalized between 6-12 months postpartum. ${ }^{6}$ Those patients with persistent cardiomegaly at the onset of next pregnancy show $60 \%$ mortality and $80 \%$ morbidity rates. ${ }^{7}$

Therefore patients with an established diagnosis of peripartum cardiomyopathy, are advocated not to conceive, because of an increased risk of recurrence with the worsening signs and symptoms. ${ }^{8}$ However if such patients come for a subsequent pregnancy, they definitely pose a challenge to their anaesthetic management. We report a case of

\section{Correspondence:}

Dr. Ayaz Farooqi,

Department of Anaesthesiology \& Critical Care,

SK Institute of Medical Sciences,Soura,Srinagar, 190011.

E-Mail: ayazkfarooqi@yahoo.co.in. peripartum cardiomyopathy requiring an emergency cesarean section which was successfully managed under an epidural anaesthesia.

\section{Methods}

A 28 year old female G2P1 was admitted in Obstetrics and Gynae department with a history of leaking for which she was planned for emergency cesarean section about 20 days ahead of her expected date of delivery.

Past history of patient revealed her first pregnancy $1 \frac{1}{2}$ year earlier, complicated by pre-eclampsia for which she had undergone LSCS and delivered a live healthy male baby.

Her past history also revealed that 40 days after her first cesarean section, she had come with the complaints of pain in left side of chest of one hour duration and was subjected to an echo and a diagnosis of peripartum cardiomyopathy was established.

The echo findings at the time of diagnosis were a global hypokinesia and mild mitral regurgitation with an ejection fraction of 35\%. Her ECG revealed sinus tachycardia. Patient was being regularly followed up in cardiology department 
where she was put on tab. Digoxin $0.25 \mathrm{mg} \mathrm{BD}$, tab. Lasilactone 1 OD (a combination of Frusemide $20 \mathrm{mg}$ and Spirinolactone $50 \mathrm{mg}$ ).

Another follow up was done in cardiology department during her second trimester where her repeat echo revealed a dilated left ventricle and borderline left ventricular dysfunction with an ejection fraction of $48 \%$. During her third trimester nearly $1 \frac{1}{2}$ month before her EDD, patient had again come with pain in the left side of chest for which cardiology consultation was sought. Tab. Betaloc (metaprolol) 12.5mg BD was added to her existing treatment.

On arrival in operating room, patient was asymptomatic on drugs with a heart rate of 90/minute and a blood pressure of $150 / 90 \mathrm{mmHg}$. On auscultation, chest was bilaterally clear with no added sounds. Her Hb was $10 \mathrm{gms}$ and BT, CT of 2'3" and 6'45" respectively. Cardiologist was requested to stay in operating room during the procedure.

Emergency cesarean section along with ligation was planned for the patient. The condition of patient as discussed with the surgeons could permit us to give epidural drug in increments. An iv access was secured with an $18 \mathrm{~g}$ peripheral venous cannula.

All the resuscitation equipment and the emergency drugs like Dopamine, Dobutamine, Nitroglycerine and Isoprenaline were kept standby. Patient was connected to a continous ECG monitor, pulse oximeter and a noninvasive blood pressure monitor.

An epidural catheter was inserted at L3-4 level in sitting position through an 18 gauge Weis-Winged needle and fixed at $11 \mathrm{cms}$ at skin. A test dose of $3 \mathrm{ml}$ of Xylocaine without Adrenaline was administered to confirm the correct placement. This was followed by $15 \mathrm{ml}$ of $0.5 \%$ Bupivacaine administered in increments to prevent any sudden heamodynamic instability. As advised by the cardiologist $20 \mathrm{mg}$ of Frusemide and $150 \mathrm{ml}$ of crystalloid were given to the patient in the beginning. The adequacy of epidural block was ensured upto T7 level. After ensuring adequacy of the block, surgeon was asked to proceed. To facilitate uterine contraction, 20 units of Oxytocin were infused slowly and additional $20 \mathrm{mg}$ of Frusemide were given after the delivery. Patient delivered a live healthy male baby with normal Apgar score and $3 \mathrm{~kg}$ weight.

There was no disturbance in heart rate or rhythm and blood pressure remained stable intra-operatively. Heart rate was maintained between $80-100 /$ minute and blood pressure remained around $130 / 80 \mathrm{mmHg}$. A top up dose of $4 \mathrm{ml}$ of $0.25 \%$ bupavacaine was administered at the time of shifting the patient.

As advised by the cardiologist, fluid intake was restricted to 1-1.5 litres per 24 hours.

On the night after surgery patient again developed chest pain radiating to left shoulder with profuse sweating. ECG showed sinus tachycardia. Cardiologist was called who gave tab.Revelol and advised to restrict the fluid only to 1 litre per
24 hours. Chest pain was relieved. Thereafter, patient remained asymptomatic with acceptable heart rate and rythm and a stable blood pressure. Patient was discharged after 8 days on cardiology follow up with the following treatment, tab. Metaprolol 25mg OD, tab. Lasilactone 1 OD and tab Digoxin $0.25 \mathrm{mg}$ OD.

\section{Discussion}

Peripartum cardiomyopathy is defined by the presence of four criteria. These include a) Development of cardiac failure in the last month of pregnancy or within five months of delivery, b) absence of an identifiable cause of cardiac failure, c) absence of any recognizable heart disease prior to last month of pregnancy, d) left ventricular systolic dysfunction demonstrated by echocardiographic criteria such as depressed ejection fraction. ${ }^{3}$ Stricter echocardiographic criteria have been recommended such as left ventricular ejection fraction of less than $45 \%$, fractional shortening of less than $30 \%$ of an M-mode echocardiographic scan or both and a left ventricular end-diastolic dimension of more than $2.7 \mathrm{~cm}$ per square metre of body surface area. ${ }^{10}$

Risk factors attributed to the development of postpartum cardiomyopathy include, multigravida, multiple gestations, pregnancy induced hypertension, pre-eclampsia and older women with multiple previous pregnancies. ${ }^{8}$

Our case fulfilled the diagnostic criteria for peripartum cardiomyopathy with the signs and symptoms developing 40 days after previous pregnancy, absence of any underlying heart disease during that period, echo findings showing global hypokinesia, left ventricular systolic dysfunction with an ejection fraction of $35 \%$. Besides, she had an associated risk factor of pre-eclamsia during her previous pregnancy.

Parturients with peripartum cardiomyopathy require special care during labour and delivery. The cardiovascular stress of labour and delivery may lead to cardiac decompensation. Anaesthesiologist may need to infuse vasoactive drugs like Nitroglycerine or Nitroprusside for preload and afterload reduction and Dopamine, Dobutamine or Milrinone for ionotropic support. ${ }^{10}$

The goals during management of anaesthesia if such patients come for cesarean section, whether elective or emergency are ${ }^{10}$ :

1. To minimize the stress of the patient.

2. Avoidance of drug induced myocardial depression.

3. Avoid increase in afterload.

4. Maintenance of normovolumia.

General anaesthetic techniques involve the use of either intravenous induction agents such as sodium thiopentone with cardio-depressent action or inhalational agents which do not gaurentee heamodynamic stability. ${ }^{8}$

Regional anaesthesia for these patients is a preferred technique and should be considered in these patients in a 
similar way as with the other types of cardiac failure. ${ }^{3}$

Subarachnoid block should be better avoided in these patients because of sudden onset of heamodynamic instability associated with it. ${ }^{8}$

Epidural anaesthesia is a preffered and better choice particularly when incremental doses of local anaesthetics are to be administered. ${ }^{8}$ In fact the sympathectomy induced afterload reduction that occurs with epidural anaesthesia can contribute to the improvement in myocardial performance in these patients. ${ }^{11}$

With an epidural catheter in place, a gradual and controlled induction of anaesthesia may improve myocardial performance and cardiac output by decreasing SVR, thus reducing the afterload on left ventricle without impairing myocardial contractility. ${ }^{8}$

Our decision to give bupavacaine in increments was to prevent any sudden heamodynamic instability. The choice of non-invasive monitoring was made keeping in view, the emergent nature of cesarean section and non handyness of the equipment and also because of concern of subjecting an otherwise asymptomatic patient, though on drugs, to the potential risks of invasive monitoring. However, a strict vigil was kept over the heamodynamic alterations, heart rate and rhythm and oxygenation of the patient with appropriate monitoring aids. Syntocinon after the delivery was used as a slow infusion to prevent sudden vasodilatation resulting in hypotension and tachycardia. This also helped in reducing the afterload and maintaining heamodynamic stability.

\section{Conclusion}

Carefully administered epidural anaesthesia is a better option for patients with peripartum cardiomyopathy undergoing cesarean section. It avoids the stress of general anaesthesia and the use of cardio-depressant drugs. It improves myocardial performance by reducing the left ventricular afterload. It is preferred over spinal anaesthesia as it prevents any sudden and rapid reduction in SVR and thereby preload which might be disastrous in low cardiac output states.
Therefore in patients with peripartum cardiomyopathy coming for cesarean section, epidural anaesthesia administered in increments is a safe and convenient technique of anaesthesia.

\section{References}

1. Lang RM, Lampert MB, Poppas A, Elkayum U. Peripheral cardiomyopathy, in: Elkayum U, Gleicher N, eds: Cardiac problems in pregnancy, 3rd ed, New York,Wiley-Lisa:1998:87-100.

2. Heider AL, Kuller JA, Strauss RA, Wells SR. Peripartum cardiomyopathy: a review of literature. Obstet Gynecol Surv 1999;54:526-31.

3. Pearson GD, Veille JC, Rahimtoola S et al. Peripartum cardiomyopathy:National Heart,Lung and Blood Institute and office of Rare diseases(National Institutes of health) Workshop recommendations and review. JAMA 2000;281:1183-88.

4. Homanx DC. Peripartum cardiomyopathy, Current concepts. NEngJ Med 1985;312:1432-37.

5. Demakis JG, Rahimtoola SH, Sutton GC et al. Natural course of peripartum cardiomyopathy. Circulation 1971; 44:1053-51.

6. Seftel H, Susser M. Maternity and myocardial failure in African women. Br HeartJ 1961:23-43.

7. Velle JC. Peripartum cardiomyopathy: A review. Am J Obstet Gynaecol 1984;148:805-18.

8. Fernando S, Prasanna A.Peripartum Cardiomyopathy (A case report). Indian Anaesthetists Forum July 2005.

9. Hibbard JU, Lindheimer M, Lang RM. A modified definition for peripartum cardiomyopathy and prognosis based on echocardiography. Obstet Gynaecol 1999;94:311-16.

10. Fragnato R. Peripartum Cardiomyopathy: A current review: SOAP(Society of Obstetric anaesthesia and Perinatology)Annual meeting, Fairmont BanffSprings, Alberta Canada, May 2007.

11. George LM, Gatt SP, Lowe S. Peripartum cardiomyopathy: four case histories and a commentary on anaesthetic management. Anaesth Intens Care 1997;25: 292-6. 